\title{
Heights of powers of Newman and Littlewood polynomials
}

\author{
by
}

\author{
Artūras Dubickas (Vilnius)
}

1. Introduction. Let $P(x)=a_{0}+a_{1} x+\cdots+a_{n} x^{n} \in \mathbb{C}[x]$, where $a_{n} \neq 0$. As usual, we say that $n=\operatorname{deg}(P)$ is the degree of $P, H(P)=\max _{0 \leq i \leq n}\left|a_{i}\right|$ is its height, and $L(P)=\sum_{i=0}^{n}\left|a_{i}\right|$ is its length. Let also $N(P)$ be the number of nonzero coefficients of $P$. Recall that $P(x)$ is called a Newman polynomial (after [18]) if its coefficients all belong to the set $\{0,1\}$. There are a variety of problems related to Newman polynomials. See, for instance, [4], [5], [6], [9], [12], [13], [20], [21] for some of them.

Recently, $\mathrm{Yu}[22]$ considered the quantity

$$
\liminf _{k \rightarrow \infty} \operatorname{deg}\left(P_{k}\right) H\left(P_{k}^{2}\right) / N\left(P_{k}\right)^{2},
$$

where $P_{k}, k=1,2, \ldots$, is a sequence of Newman polynomials with $\operatorname{deg}\left(P_{1}\right)<$ $\operatorname{deg}\left(P_{2}\right)<\cdots$. He conjectured (see Conjecture 2 in [22]) that this limit is always at least 1 if $N\left(P_{k}\right) / \operatorname{deg}\left(P_{k}\right) \rightarrow 0$ as $k \rightarrow \infty$. This conjecture, if proved, would give a sharp bound for the so-called $B_{2}[g]$ sets which generalize classical Sidon sets $B_{2}$ [1]. (See Section 4 for some definitions and a discussion concerning $B_{h}[g]$ sets.) Berenhaut and Saidak [2] proved that the condition $\lim _{k \rightarrow \infty} N\left(P_{k}\right) / \operatorname{deg}\left(P_{k}\right)=0$ is indeed necessary in this conjecture. More precisely, they showed that there is a sequence of Newman polynomials for which the above limit is $8 / 9$. (In fact, they obtained this limit by considering the sequence of Newman polynomials $P_{k}(x)=1+x+\cdots+x^{k-1}+x^{2 k}+$ $\left.x^{2 k+1}+\cdots+x^{4 k}, k=1,2, \ldots\right)$ In conclusion, they asked whether $8 / 9$ is the smallest possible limit, and, if no, how small the limit can be. Below, we shall give a sequence of Newman polynomials with a limit $5 / 6$, thus answering this question in the negative. Moreover, they asked what happens for higher powers of Newman polynomials and also for Littlewood polynomials, i.e., those with coefficients in $\{-1,1\}$. This paper is also devoted to the study of these questions.

2000 Mathematics Subject Classification: 11C08, 30C10.

Key words and phrases: Newman polynomial, Littlewood polynomial, height, Legendre symbol. 
For any fixed positive integer $m$, we shall consider the following quantity:

$$
Q_{m}(P)=(\operatorname{deg}(P)+1) H\left(P^{m}\right) / N(P)^{m} .
$$

Obviously, the fact that $\operatorname{deg}(P)+1$ is taken instead of $\operatorname{deg}(P)$ does not make any difference to the above problem for $m=2$, because $\operatorname{deg}(P) /(\operatorname{deg}(P)+1)$ $\rightarrow 1$ as $\operatorname{deg}(P) \rightarrow \infty$. So we can restate the problem considered in [2] as follows: find the smallest possible limit $\liminf _{k \rightarrow \infty} Q_{2}\left(P_{k}\right)$ over all sequences of Newman polynomials $P_{k}, k=1,2, \ldots$, satisfying $\operatorname{deg}\left(P_{1}\right)<\operatorname{deg}\left(P_{2}\right)<\cdots$.

In the next section we state our main results. Their proofs will be given in Section 3. Some further examples and an additional motivation for the study of the quantity $Q_{m}(P)$ which was introduced above will be given in Section 4 .

2. Results. Note that, for any positive integer $m$, and any Newman polynomial $P$, we have

$$
Q_{m}(P) \geq 1 / m
$$

Indeed, let $P(x)=a_{0}+a_{1} x+\cdots+a_{n} x^{n}$ be a Newman polynomial of degree $n$. Since $a_{i} \in\{0,1\}$ for each $i=0,1, \ldots, n$, we have

$$
\begin{aligned}
N(P)^{m} & =P(1)^{m}=L(P)^{m}=L\left(P^{m}\right) \\
& \leq\left(\operatorname{deg}\left(P^{m}\right)+1\right) H\left(P^{m}\right)=(n m+1) H\left(P^{m}\right) .
\end{aligned}
$$

Hence $Q_{m}(P)=(n+1) H\left(P^{m}\right) / N(P)^{m} \geq(n+1) /(n m+1) \geq 1 / m$, as claimed.

This bound is sharp for $m=1$, because $Q_{1}\left(1+x+\cdots+x^{n}\right)=1$ for any nonnegative integer $n$. It seems likely that this bound is not sharp for $m \geq 2$ (see Section 4).

Our next theorem explains the advantage of considering the factor $\operatorname{deg}(P)$ +1 instead of $\operatorname{deg}(P)$ in the definition of $Q_{m}(P)$.

Theorem 1. Let $m \geq 2$ be a fixed integer, and let $P$ be a Newman polynomial. Then there is a sequence of Newman polynomials $P_{k}, k=1,2, \ldots$, with increasing degrees such that $Q_{m}\left(P_{k}\right) \leq Q_{m}(P)$ for each $k \geq 1$. Moreover, for $m=2$, there is a sequence of Newman polynomials $P_{k}, k=1,2, \ldots$, with increasing degrees such that $Q_{2}\left(P_{k}\right)=Q_{2}(P)$ for each positive integer $k$.

By Theorem 1, instead of working with sequences of Newman polynomials $P_{1}, P_{2}, \ldots$ with increasing degrees and with $\liminf _{k \rightarrow \infty} Q_{m}\left(P_{k}\right)$ small, it is sufficient to find a single Newman polynomial $P$ with $Q_{m}(P)$ small. Then Theorem 1 guarantees that there is a sequence of Newman polynomials $P_{1}, P_{2}, \ldots$ with increasing degrees such that

$$
\liminf _{k \rightarrow \infty} Q_{m}\left(P_{k}\right) \leq Q_{m}(P) .
$$


The problem studied in [2] and [22] thus reduces to finding the infimum of the quantity $Q_{2}(P)$, where $P$ runs over all Newman polynomials. More generally, for higher powers, one may look for

$$
\inf _{P \text { Newman }} Q_{m}(P)
$$

where $m \geq 2$ is a fixed integer.

Obviously, $Q_{m}(P)=Q_{m}\left(P^{*}\right)$, where $P(0) \neq 0$ and $P^{*}(x)=x^{\operatorname{deg} P} P(1 / x)$ is a polynomial reciprocal to $P$. So, if there is a polynomial $P$ at which the infimum $\inf _{P \text { Newman }} Q_{m}(P)$ is attained, then this $P$ is not unique.

In particular, for $m=2$, let us take $P(x)=1+x+x^{3}$. Then $\operatorname{deg}(P)=$ $N(P)=3, H\left(P^{2}\right)=H\left(1+2 x+x^{2}+2 x^{3}+2 x^{4}+x^{6}\right)=2$, giving $Q_{2}(P)=8 / 9$. Combined with Theorem 1 this implies the main result of [2]. Note that the polynomial $1+x^{2}+x^{3}$ (which is reciprocal to $1+x+x^{3}$ ) already features as extremal in the following well-known problem: find $f(n)=$ $\sup _{N(P)=n, P \text { Newman }} \inf _{|z|=1}|P(z)|$. It is shown in [6] that $f(3)$ is attained at the polynomial $1+x^{2}+x^{3}$. It seems that this is a mere coincidence, because the polynomial $1+x^{2}+x^{3}+x^{4}$ maximizing $f(4)$ (see [13]) is of no use in the study of "small" $Q_{m}(P)$.

The value $8 / 9$ is not the smallest value attained by $Q_{2}(P)$, where $P$ is a Newman polynomial. We have computed $Q_{2}(P)$ for all Newman polynomials of degree at most 20 . The smallest value is obtained for the polynomial

$$
P(x)=1+x+x^{2}+x^{3}+x^{4}+x^{5}+x^{8}+x^{9}+x^{12}+x^{14}+x^{18}+x^{19}
$$

of degree 19 with 12 nonzero coefficients. Since $H\left(P^{2}\right)=6$, we find that $Q_{2}(P)=20 \cdot 6 / 12^{2}=5 / 6$. Of course, this answers the question raised in $[2]$ in the negative. Combined with Theorem 1, this example shows that the limit considered in [22] and [2] can be at least as small as 5/6. This limit is attained by the sequence of polynomials $P_{k}(x)=\left(1+x+\cdots+x^{k-1}\right) P\left(x^{k}\right)$ of degree $20 k-1$, where $k=1,2, \ldots$, since $Q_{2}\left(P_{k}\right)=5 / 6$ for each positive integer $k$ (see the proof of Theorem 1 ).

We also calculated the minimal value of $Q_{m}(P)$, where $P$ is a Newman polynomial of degree $\leq 20$, for each $m \in\{2,3,4,5,6\}$. The extremal polynomials $P$ (to be precise, one of them, since there are at least two if $P$ is not reciprocal) with corresponding values for $Q_{m}(P)$ are given in the following table:

\begin{tabular}{rrcrrr}
\hline$m$ & \multicolumn{1}{c}{$P$} & $\operatorname{deg} P$ & $N(P)$ & $H\left(P^{m}\right)$ & \multicolumn{1}{c}{$Q_{m}(P)$} \\
\hline 2 & 11000101001100111111 & 19 & 12 & 6 & $5 / 6=0.83333333 \ldots$ \\
3 & 111011100011010011111 & 20 & 14 & 84 & $9 / 14=0.64285714 \ldots$ \\
4 & 11010001010001101111 & 19 & 11 & 404 & $8080 / 14641=0.55187487 \ldots$ \\
5 & 101010001000010000111 & 20 & 8 & 750 & $7875 / 16384=0.48065185 \ldots$ \\
6 & 101010000010000011 & 17 & 6 & 1110 & $185 / 432=0.42824074 \ldots$ \\
\hline
\end{tabular}


In this table, the polynomial is given by the string of its coefficients in descending order. For instance, the string 101010000010000011 corresponds to the Newman polynomial $P(x)=1+x+x^{7}+x^{13}+x^{15}+x^{17}$. We have $N(P)=6, H\left(P^{6}\right)=1110$, where 1110 is the coefficient of $x^{53}$ in $P^{6}$, so $Q_{6}(P)=18 \cdot 1110 / 6^{6}=185 / 432$. According to our computations, this polynomial gives the minimal value for $Q_{6}(P)$, where $P$ runs over all Newman polynomials of degree at most 20 .

For a Littlewood polynomial $P$ (with coefficients \pm 1 ), we have $N(P)=$ $\operatorname{deg}(P)+1$, so

$$
Q_{m}(P)=H\left(P^{m}\right) /(\operatorname{deg}(P)+1)^{m-1} .
$$

Since $H(P)=1$, we obtain $Q_{1}(P)=1$ for every Littlewood polynomial $P$.

In our next theorem we construct a sequence of Littlewood polynomials $P_{1}, P_{2}, P_{3}, \ldots$ with increasing degrees such that $\lim _{k \rightarrow \infty} Q_{2}\left(P_{k}\right)=0$. For any integer $a$ and any prime number $p$, we denote by $\left(\frac{a}{p}\right)$ the Legendre symbol which is equal to 0 if $p \mid a$ and, if $a$ and $p$ are coprime, it is equal to 1 or -1 depending on whether or not the congruence $X^{2} \equiv a(\bmod p)$ has an integer solution.

TheOREM 2. Let $k \geq 2$ be a fixed positive integer, and let $p$ be a prime number satisfying $2 k+3 \leq p<4 k+6$. Then $P_{k}(x)=\sum_{i=0}^{k}\left(\frac{i+1}{p}\right) x^{i}$ is a Littlewood polynomial which satisfies $H\left(P_{k}^{2}\right)<c \sqrt{k} \log k$, where $c$ is an absolute positive constant.

From this theorem, we will derive that

$$
\inf _{P \text { Littlewood }} Q_{m}(P)=0
$$

for any integer $m \geq 2$.

Corollary 3. Let $m \geq 2$ be a fixed positive integer. Then there is a sequence of Littlewood polynomials $P_{k}, k=1,2, \ldots$, with increasing degrees such that $\lim _{k \rightarrow \infty} Q_{m}\left(P_{k}\right)=0$.

The idea of using Fekete type polynomials (whose coefficients are Legendre or Jacobi symbols as in Theorem 2) in this kind of problems is not new. Such polynomials are known to give "large" asymptotic merit factor, that is, the quantity $\operatorname{MF}(P)=\|P\|_{2}^{4} /\left(\|P\|_{4}^{4}-\|P\|_{2}^{4}\right)$, where $\|P\|_{s}=$ $\left((2 \pi)^{-1} \int_{0}^{2 \pi}\left|P\left(e^{i \theta}\right)\right|^{s} d \theta\right)^{1 / s}, i=\sqrt{-1}$. See, for instance, [14] and [3].

Note that $\|P\|_{2}=\sqrt{k+1}$ for any Littlewood (and, more generally, unimodular) polynomial $P$ of degree $k$. Writing $P(x)^{2}=\sum_{j=0}^{2 k} A_{j} x^{j}$, we have $\|P\|_{4}=\left(\sum_{j=0}^{2 k} A_{j}^{2}\right)^{1 / 4}$. As the norm $\|P\|_{s}$ is nondecreasing in $s$ (for any fixed $P(x) \in \mathbb{C}[x])$, we deduce that

$$
\sqrt{k+1}=\|P\|_{2} \leq\|P\|_{4} \leq\left((2 k+1) \max _{0 \leq j \leq 2 k} A_{j}^{2}\right)^{1 / 4}=\left((2 k+1) H\left(P^{2}\right)^{2}\right)^{1 / 4} .
$$


Squaring the last inequality yields

$$
H\left(P^{2}\right) \geq \sqrt{(k+1) / 2}
$$

for any Littlewood (and any unimodular) polynomial $P$ of degree $k$. Compare to Theorem 2, where there is an extra factor $\log k$.

It seems likely that there is a sequence of Littlewood polynomials $P_{k}$, $k=1,2, \ldots$, where $\operatorname{deg} P_{k}=k$, such that $H\left(P_{k}^{2}\right)<c \sqrt{k}$ with an absolute positive constant $c$. This would give a sharp (up to a constant) version of Theorem 2. Such an improvement would be related to the still open conjecture of Littlewood on flat polynomials on the unit circle claiming that, for any $k$, there exist Littlewood polynomials $P_{k}$ of degree $k$ such that

$$
c_{1} \sqrt{k+1}<\left|P_{k}(z)\right|<c_{2} \sqrt{k+1}
$$

on $|z|=1$ with some absolute positive constants $c_{1}$ and $c_{2}$. A version of this problem for polynomials $P_{k}$ with unimodular coefficients was settled by Kahane [16] (with $c_{1}=1-\varepsilon$ and $c_{2}=1+\varepsilon$ for $k$ large enough). Beck [1] proved Littlewood's conjecture for polynomials whose coefficients are $\ell$ th roots of unity, where $\ell=400$.

3. Proofs. For the proof of Theorem 1 we need the following lemma.

Lemma 4. Let $k, m$ be two positive integers, and let

$$
\left(1+x+x^{2}+\cdots+x^{k-1}\right)^{m}=b_{0}+b_{1} x+\cdots+b_{m(k-1)} x^{m(k-1)} .
$$

Set

$$
B_{j}=b_{j}+b_{j+k}+b_{j+2 k}+\cdots .
$$

Then $B_{j}=k^{m-1}$ for each $j=0,1, \ldots, k-1$.

Proof. Let $\mu$ be a primitive $k$ th root of unity. Since $1+x+\cdots+x^{k-1}=$ $\left(x^{k}-1\right) /(x-1)$ vanishes at $x=\mu^{i}$, where $i=1, \ldots, k-1$, and is equal to $k$ at $x=1$, we deduce that $B_{0}+B_{1} \mu^{i}+\cdots+B_{k-1} \mu^{i(k-1)}=0$ for $i=1, \ldots, k-1$ and $B_{0}+B_{1}+\cdots+B_{k-1}=k^{m}$. This linear system of $k$ equations in $k$ unknowns $B_{0}, B_{1}, \ldots, B_{k-1}$ has a unique solution, because the corresponding Vandermonde determinant is nonzero. On the other hand, $B_{0}=B_{1}=\cdots=B_{k-1}=k^{m-1}$ is a solution of this system. Hence it is the only solution. This completes the proof of the lemma.

Proof of Theorem 1. Given a Newman polynomial $P(x)$, we set

$$
P_{k}(x)=\left(1+x+x^{2}+\cdots+x^{k-1}\right) P\left(x^{k}\right)
$$

for $k=1,2, \ldots$. It is clear that each $P_{k}$ is a Newman polynomial of degree $\operatorname{deg}\left(P_{k}\right)=k \operatorname{deg}(P)+k-1$. Thus $\operatorname{deg}\left(P_{1}\right)<\operatorname{deg}\left(P_{2}\right)<\cdots$. Furthermore, from the definition of $P_{k}$ it follows that $N\left(P_{k}\right)=N(P) k$. Hence $Q_{m}\left(P_{k}\right)=$ $\left(\operatorname{deg}\left(P_{k}\right)+1\right) H\left(P_{k}^{m}\right) / N\left(P_{k}\right)^{m}=(\operatorname{deg}(P)+1) H\left(P_{k}^{m}\right) /\left(N(P)^{m} k^{m-1}\right)$, giving

$$
Q_{m}\left(P_{k}\right)=Q_{m}(P) H\left(P_{k}^{m}\right) /\left(H\left(P^{m}\right) k^{m-1}\right) \text {. }
$$


It remains to prove that $H\left(P_{k}^{m}\right) \leq H\left(P^{m}\right) k^{m-1}$ for positive integers $k$ and $m$, where equality holds for $m=1$ and $m=2$. (Clearly, for $m=1$, we have $H\left(P_{k}\right)=H(P)=1$.)

For $m \geq 2$, by Lemma 4 , we write

$$
\left(1+x+\cdots+x^{k-1}\right)^{m}=b_{0}+b_{1} x+\cdots+b_{m(k-1)} x^{m(k-1)} .
$$

Setting

$$
P(x)^{m}=c_{0, m}+c_{1, m} x+\cdots+c_{m n, m} x^{m n},
$$

where $n=\operatorname{deg}(P)$, we obtain

$$
\begin{aligned}
P_{k}(x)^{m} & =\left(b_{0}+b_{1} x+\cdots+b_{m(k-1)} x^{m(k-1)}\right)\left(c_{0, m}+c_{1, m} x^{k}+\cdots+c_{m n, m} x^{m n k}\right) \\
& =\sum_{s=0}^{m(n k+k-1)} h_{s} x^{s} .
\end{aligned}
$$

Here $h_{s}=\sum_{i+k j=s} b_{i} c_{j, m}$, where $0 \leq i \leq m(k-1)$ and $0 \leq j \leq m n$. Set $b_{i}=0$ for $i>m(k-1)$ and $c_{j, m}=0$ for $j>m n$. Then

$$
h_{s}=b_{s} c_{0, m}+b_{s-k} c_{1, m}+b_{s-2 k} c_{2, m}+\cdots+b_{s-[s / k] k} c_{[s / k], m} .
$$

(Here and below, [...] stands for the integer part.) Now, since all $b_{i}$ and all $c_{j, m}$ are nonnegative integers, using Lemma 4 , we obtain

$$
\begin{aligned}
\left|h_{s}\right|=h_{s} & \leq\left(b_{s}+b_{s-k}+\cdots+b_{s-[s / k] k}\right) \max _{0 \leq j \leq[s / k]} c_{j, m} \leq k^{m-1} \max _{0 \leq j \leq m n}\left|c_{j, m}\right| \\
& =k^{m-1} H\left(P^{m}\right) .
\end{aligned}
$$

This implies that $H\left(P_{k}^{m}\right) \leq H\left(P^{m}\right) k^{m-1}$, as claimed.

In particular, for $m=2$, we have

$$
\begin{aligned}
& \left(1+x+x^{2}+\cdots+x^{k-1}\right)^{2} \\
& \quad=1+2 x+3 x^{2}+\cdots+k x^{k-1}+(k-1) x^{k-2}+\cdots+2 x^{2 k-3}+x^{2 k-2} .
\end{aligned}
$$

Let $j$ be an index satisfying $c_{j, 2}=H\left(P^{2}\right)$. It follows that the coefficient of $x^{k-1+j}$ in $P_{k}(x)^{2}=\left(1+x+\cdots+x^{k-1}\right)^{2} P\left(x^{k}\right)^{2}$ is greater than or equal to $k H\left(P^{2}\right)$. Hence $H\left(P_{k}^{2}\right) \geq H\left(P^{2}\right) k$. On the other hand, setting $m=2$ in the inequality $H\left(P_{k}^{m}\right) \leq H\left(P^{m}\right) k^{m-1}$, which is already proved, we obtain $H\left(P_{k}^{2}\right) \leq H\left(P^{2}\right) k$. Thus $H\left(P_{k}^{2}\right)=H\left(P^{2}\right) k$ for each positive integer $k$. This completes the proof of the theorem.

In the proof of Theorem 2 we shall need an upper bound for an incomplete sum of multiplicative characters. The following lemma is extracted from Theorem 2 in [17]:

Lemma 5. Let $p \geq 3$ be a fixed prime, and let $\chi$ be a multiplicative character modulo $p$ of order $m \geq 2$. Suppose that $M$ and $K$ are integers with $1 \leq K<p$. If $f(x)$ is a polynomial in $\mathbb{F}_{p}[x]$ with d distinct roots which 
is not the mth power then

$$
\left|\sum_{j=M+1}^{M+K} \chi(f(j))\right|<C d \sqrt{p} \log p,
$$

where $C>0$ is an absolute constant.

In particular, for the Legendre symbol, which is a multiplicative character of order 2 , by taking $f(x)=x^{2}-l x-l-1=(x+1)(x-l-1)$, we obtain

$$
\left|\sum_{j=M+1}^{M+K}\left(\frac{j^{2}-l j-l-1}{p}\right)\right|<2 C \sqrt{p} \log p
$$

for any prime number $p \geq 3$ and any integer $l$ satisfying $0 \leq l<p-2$. Indeed, then $f(x)$ is not a square, because its roots -1 and $l+1$ treated as elements of the field $\mathbb{F}_{p}$ are distinct. So the above inequality follows from Lemma 5.

Proof of Theorem 2. None of the numbers $1, \ldots, k+1$ is divisible by $p$, so $P_{k}(x)=\sum_{i=0}^{k}\left(\frac{i+1}{p}\right) x^{i}$ is indeed a Littlewood polynomial. Its square is equal to $P_{k}(x)^{2}=\sum_{l=0}^{2 k} g_{l} x^{l}$, where, by the multiplicative property of the Legendre symbol and $\left(\frac{-1}{p}\right)=(-1)^{(p-1) / 2}$, we have

$$
\begin{aligned}
g_{l} & =\sum_{j+t=l}\left(\frac{j+1}{p}\right)\left(\frac{t+1}{p}\right)=\sum_{j+t=l}\left(\frac{j t+j+t+1}{p}\right) \\
& =\sum_{j=\max \{l-k, 0\}}^{\min \{l, k\}}\left(\frac{j(l-j)+l+1}{p}\right)=(-1)^{(p-1) / 2} \sum_{j=\max \{l-k, 0\}}^{\min \{l, k\}}\left(\frac{j^{2}-l j-l-1}{p}\right) .
\end{aligned}
$$

Now, since $l \leq 2 k$ and $2 k+3 \leq p$, we have $l<p-2$. Hence $\min \{l, k\}-$ $\max \{l-k, 0\}+1<p$, and one can apply the estimate given below Lemma 5 which yields $H\left(P_{k}^{2}\right)=\max _{0 \leq l \leq 2 k}\left|g_{l}\right|<2 C \sqrt{p} \log p$. Consequently, for each $k \geq 2$, using $p<4 k+6$, we obtain

$$
H\left(P_{k}^{2}\right)<2 C \sqrt{4 k+6} \log (4 k+6)<c \sqrt{k} \log k,
$$

where $c$ is an absolute positive constant.

Proof of Corollary 3. It is sufficient to prove that, for the sequence of polynomials $P_{2}, P_{3}, \ldots$, where $P_{k}(x)=\sum_{i=0}^{k}\left(\frac{i+1}{p}\right) x^{i}$ and $p$ is a prime number satisfying $2 k+3 \leq p<4 k+6$, we have $\lim _{k \rightarrow \infty} Q_{m}\left(P_{k}\right)=0$.

Note that

$$
Q_{m}\left(P_{k}\right)=H\left(P_{k}^{m}\right)(k+1)^{1-m}
$$

for each $m \geq 2$, because $P_{k}$ is a Littlewood polynomial of degree $k$. Using the inequalities $H(P Q) \leq L(P) H(Q)$ and $L(P Q) \leq L(P) L(Q)$, which hold 
for any complex polynomials $P$ and $Q$, we obtain

$$
H\left(P_{k}^{m}\right)=H\left(P_{k}^{m-2} P_{k}^{2}\right) \leq L\left(P_{k}^{m-2}\right) H\left(P_{k}^{2}\right) \leq L\left(P_{k}\right)^{m-2} H\left(P_{k}^{2}\right) .
$$

Next, by Theorem $2, H\left(P_{k}^{2}\right)<c \sqrt{k} \log k$. Evidently, $L\left(P_{k}\right)=k+1$. Hence $H\left(P_{k}^{m}\right)<(k+1)^{m-2} c \sqrt{k} \log k<c(k+1)^{m-3 / 2} \log k$. This implies that

$$
0<Q_{m}\left(P_{k}\right)=H\left(P_{k}^{m}\right)(k+1)^{1-m}<c(k+1)^{-1 / 2} \log k,
$$

giving $\lim _{k \rightarrow \infty} Q_{m}\left(P_{k}\right)=0$.

4. Examples and sumset related problems. Let us consider the Newman polynomial $P(x)=1+x$. Then $\operatorname{deg}(P)=1, N(P)=2, H\left(P^{m}\right)=$ $\left(\begin{array}{c}m \\ {[m / 2]}\end{array}\right)$, so

$$
Q_{m}(1+x)=2^{1-m}\left(\begin{array}{c}
m \\
{[m / 2]}
\end{array}\right) .
$$

By Stirling's formulae, $\left(\begin{array}{c}m \\ {[m / 2]}\end{array}\right)=m ! /([m / 2] !(m-[m / 2]) !) \sim 2^{m+1} / \sqrt{2 \pi m}$ as $m \rightarrow \infty$. Thus $Q_{m}(1+x) \sim 4 / \sqrt{2 \pi m}$ as $m \rightarrow \infty$. Combined with the lower bound for $Q_{m}(P)$, this shows that

$$
1 / m \leq \inf _{P \text { Newman }} Q_{m}(P) \leq \sqrt{8 / \pi m}+\varepsilon
$$

for any positive number $\varepsilon$ and any fixed integer $m \geq m(\varepsilon)$.

The problem of minimizing $Q_{m}(P)$ has applications to sumset related problems and vice versa this quantity can be studied via such problems. Take, for instance, the Newman polynomial $P(x)=1+x^{r_{1}}+\cdots+x^{r_{n-1}}$, where $0=r_{0}<r_{1}<\cdots<r_{n-1}=D$ and $n \geq 2$. Its degree is $\operatorname{deg}(P)=$ $r_{n-1}=D$. Clearly, $P$ has $n$ nonzero coefficients, so $N(P)=n$. It is easy to see that $H\left(P^{2}\right)=2$ if and only if all $n(n+1) / 2$ sums $r_{i}+r_{j}$, where $0 \leq i \leq j \leq n-1$, are distinct. The set $\left\{r_{0}, r_{1}, \ldots, r_{n-1}\right\}$ with this property is called a Sidon set. The cardinality $n$ of the largest Sidon set in $\{0,1, \ldots, D\}$ is known to be of the order $\sqrt{D}+O\left(D^{c}\right)$, where $0<c<1 / 2$, as $D \rightarrow \infty$ (see [10], [11]). This implies that, for any $\varepsilon>0$, the inequality $Q_{2}(P)>2-\varepsilon$ holds for any Newman polynomial $P$ of sufficiently large degree satisfying $H\left(P^{2}\right)=2$.

More generally, one can consider the sets $\mathcal{A} \subset\{1, \ldots, D\}$ such that every integer $n$ has at most $g$ distinct representations as $n=a_{1}+\cdots+a_{h}$ with $a_{j} \in \mathcal{A}$. Unfortunately, different authors define $B_{h}[g]$ sets in a different way. Below, we follow the notation of O'Bryant [19]. He defines a sequence $\mathcal{A}$ to be a $B_{h}^{*}[g]$ sequence if the coefficients of $\left(\sum_{a \in \mathcal{A}} x^{a}\right)^{h}$ are bounded above by $g$. Then a $B_{h}[g]$ sequence is defined as a $B_{h}^{*}[h ! g]$ sequence, and $R_{h}(g, D)$ is defined as the largest cardinality of a $B_{h}^{*}[g]$ sequence contained in $\{1, \ldots, D\}$. With this notation, since $P(x)=x^{-1} \sum_{a \in \mathcal{A}} x^{a}$ is a Newman polynomial of degree $\leq D-1$, we have $Q_{h}(P) \leq D g / R_{h}(g, D)^{h}$. The bound $Q_{h}(P) \geq c_{h}$ 
is thus equivalent to $R_{h}(g, D) /(D g)^{1 / h} \leq c_{h}^{-1 / h}$. In particular, the question of whether $Q_{2}(P) \geq c_{2}$ (where, by our example of degree 19 polynomial, $c_{2}$ is known to be $\leq 5 / 6$ ) for any Newman polynomial $P$ is equivalent to the question of whether $R_{2}(g, D) / \sqrt{D g} \leq c_{2}^{-1 / 2}$ for any $B_{2}^{*}[g]$ set contained in $\{1, \ldots, D\}$. See, for instance, the papers [7], [8], [15], [22] and the survey paper [19] for various inequalities concerning upper and lower bounds for the cardinality of $B_{h}[g]$ sets contained in $\{1, \ldots, D\}$.

Using the definitions of $B_{2}[g]$ and $B_{2}^{*}[g]$ sets given above, from the literature quoted in [19], one can easily derive bounds for the cardinality of, say, $B_{2}^{*}[g]$ sets and via them bounds for $Q_{2}(P)$. Usually, these bounds hold under some additional restrictions on the set of Newman polynomials. For example, one of the results of Green [15], rewritten in the terminology of Newman polynomials, implies that $Q_{2}(P)>0.7619$ for any Newman polynomial $P$ of sufficiently large degree satisfying $H\left(P^{2}\right)=4$. The main result of $\mathrm{Yu}[22]$ is equivalent to the inequality $Q_{2}(P)>0.625$ for any Newman polynomial $P$ such that $H\left(P^{2}\right)$ is fixed and $\operatorname{deg}(P)$ is large enough. Recall that his conjecture (Conjecture 2 in [22]) asserts that for any $\varepsilon>0$ there is a $\delta>0$ such that $Q_{2}(P)>1-\varepsilon$ for any Newman polynomial $P$ satisfying $N(P)<\delta \operatorname{deg}(P)$.

This research was supported by INTAS grant no. 03-51-5070. I thank Jonas Jankauskas who performed the calculations presented in the table.

\section{References}

[1] J. Beck, Flat polynomials on the unit circle — note on a problem of Littlewood, Bull. London Math. Soc. 23 (1991), 269-277.

[2] K. S. Berenhaut and F. Saidak, A note on the maximal coefficients of squares of Newman polynomials, submitted.

[3] P. Borwein and K.-K. S. Choi, Explicit merit factor formulae for Fekete and Turyn polynomials, Trans. Amer. Math. Soc. 354 (2002), 219-234.

[4] P. Borwein and M. J. Mossinghoff, Newman polynomials with prescribed vanishing and integer sets with distinct subset sums, Math. Comp. 72 (2003), 787-800.

[5] D. W. Boyd, Large Newman polynomials, in: Diophantine Analysis (Kensigton, 1985), London Math. Soc. Lecture Note Ser. 109, Cambridge Univ. Press, Cambridge, 1986, 159-170.

[6] D. M. Campbell, H. R. P. Ferguson and R. W. Forcade, Newman polynomials on $|z|=1$, Indiana Univ. Math. J. 32 (1983), 517-525.

[7] J. Cilleruelo and J. Jiménez-Urroz, $B_{h}[g]$ sequences, Mathematika 47 (2000), 109 115.

[8] J. Cilleruelo, I. Ruzsa and C. Trujillo, Upper and lower bounds for finite $B_{h}[g]$ sequences, J. Number Theory 97 (2002), 26-34.

[9] T. Erdélyi, Extremal properties of the derivatives of the Newman polynomials, Proc. Amer. Math. Soc. 131 (2003), 3129-3134. 
[10] P. Erdős, On a problem of Sidon in additive number theory and some related problems. Addendum, J. London Math. Soc. 19 (1944), 208.

[11] P. Erdős and P. Turán, On a problem of Sidon in additive number theory and some related problems, ibid. 16 (1941), 212-215.

[12] M. Filaseta and M. Matthews Jr., On the irreducibility of 0,1-polynomials of the form $f(x) x^{n}+g(x)$, Colloq. Math. 99 (2004), 1-5.

[13] B. Goddard, Finite exponential series and Newman polynomials, Proc. Amer. Math. Soc. 116 (1992), 313-320.

[14] M. J. Golay, The merit factor of Legendre sequences, IEEE Trans. Inform. Theory 29 (1983), 934-936.

[15] B. Green, The number of squares and $B_{h}[g]$ sets, Acta Arith. 100 (2001), 365-390.

[16] J.-P. Kahane, Sur les polynômes à coefficients unimodulaires, Bull. London Math. Soc. 12 (1980), 321-342.

[17] C. Mauduit and A. Sárközy, On finite pseudorandom binary sequences. I: Measure of pseudorandomness, the Legendre symbol, Acta Arith. 82 (1997), 365-377.

[18] D. J. Newman, An $L^{1}$ extremal problem for polynomials, Proc. Amer. Math. Soc. 16 (1965), 1287-1290.

[19] K. O'Bryant, A complete annotated bibliography of work related to Sidon sequences, Electronic J. Combin. Dynamical Surveys DS11 (2004), 39 pp.

[20] A. M. Odlyzko and B. Poonen, Zeros of polynomials with 0, 1 coefficients, Enseign. Math. (2) 39 (1993), 317-348.

[21] C. J. Smyth, Some results on Newman polynomials, Indiana Univ. Math. J. 34 (1985), 195-200.

[22] G. Yu, An upper bound for $B_{2}[g]$ sets, J. Number Theory 122 (2007), 211-220.

Department of Mathematics and Informatics

Vilnius University

Naugarduko 24

Vilnius LT-03225, Lithuania

E-mail: arturas.dubickas@mif.vu.lt

Received on 26.10.2006

and in revised form on 27.12.2006 